\title{
Design of HVDC Supplementary Controller Based on Extension Control Strategy
}

\author{
Yong $\mathrm{He}^{1, \mathrm{a}}$, Xiaoxiang Zhao ${ }^{1, \mathrm{~b}}$ and Mingdong Wang ${ }^{1, \mathrm{c}}$ \\ ${ }^{1}$ School of Electrical Engineering, Zhengzhou University, Zhengzhou 450001, China \\ ahey1989@126.com, b'zhaoxiaoxiang@zzu.edu.cn, cwangmingdong@zzu.edu.cn
}

\begin{abstract}
Keywords: high voltage direct current (HVDC); supplementary controller; extension control; frequency stability

Abstract: A HVDC supplementary controller is designed based on Extension control method, the controller can be divided into different feature status depending on the magnitude and speed of the system frequency change, to take a different calculation method to control the output in different measurement modes quickly and effectively modulating the DC power to achieve a power balancing system. The time-domain simulation on the four machine two area system by PSCAD/EMTDC verifies the effectiveness of the designed controller.
\end{abstract}

\section{Introduction}

High voltage direct current (HVDC) transmission has a unique advantage in long distance and large capacity transmission, with the development and delivery of distance water, thermal power base, HVDC transmission has become the main way of large capacity, long distance transmission[1]. Sharing a large proportion of the power capacity, the DC link long distance transmission of massive power has great influence on the normal operation of the grid and accident condition of isolated operation. As DC converter station is a load not sensitive to the grid frequency in terms of sending-end grid, how to use the controls to maintain the appropriate DC transmission side grid frequency stability is worthy of study. The power of DC project can be increased or decreased rapidly, also can be adjusted according to the predetermined trend of various modulation function[2]. According to the highly controllable features of HVDC, we can use the AC / DC system combined regulation means to improve system stability. To achieve such a request, the HVDC supplementary controller can be added to the original control strategy of HVDC converter station.

In this paper, a HVDC supplementary controller is designed based on Extension control method, the controller can be divided into different feature status depending on the magnitude and speed of the system frequency change, to take a different calculation method to control the output in different measurement modes quickly and effectively modulating the DC power to achieve a power balancing system. This controller has fast response speed and high regulation efficiency, also, the time-domain simulation on the four machine two area system by PSCAD/EMTDC verifies the effectiveness of the designed controller.

\section{The Design Principle of Extension Control}

Extension control method is to deal with control problems from the perspective of information conversion, making the controlled variable converted from unqualified range to qualified range, so that the control effect transformed from dissatisfied to satisfied[3]. The structure of extension controller is shown in Figure 1, and the controller consists feature extraction, correlation calculation, pattern recognition and control output of four parts.

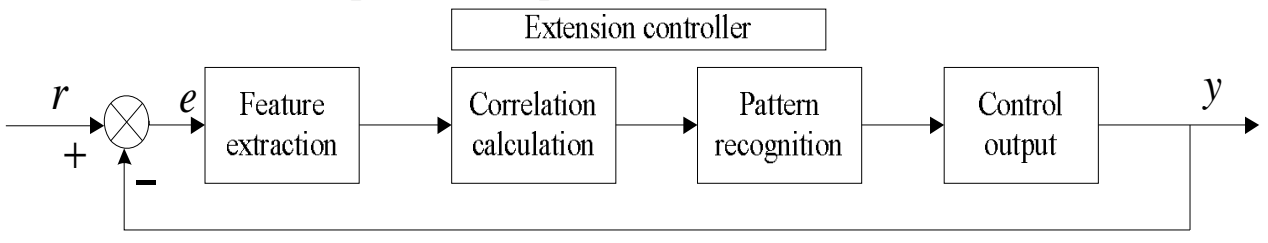

Fig.1 The structure of extension controller 


\section{Feature Extraction}

According to the literature [4], using the deviation $e$ and deviation differential $\&_{\mathrm{as}}$ the feature quantity of the system, so the characteristic state $S(e$, \&) of the extension set can be represented in Figure 2, and the shaded part represents the classical domain.

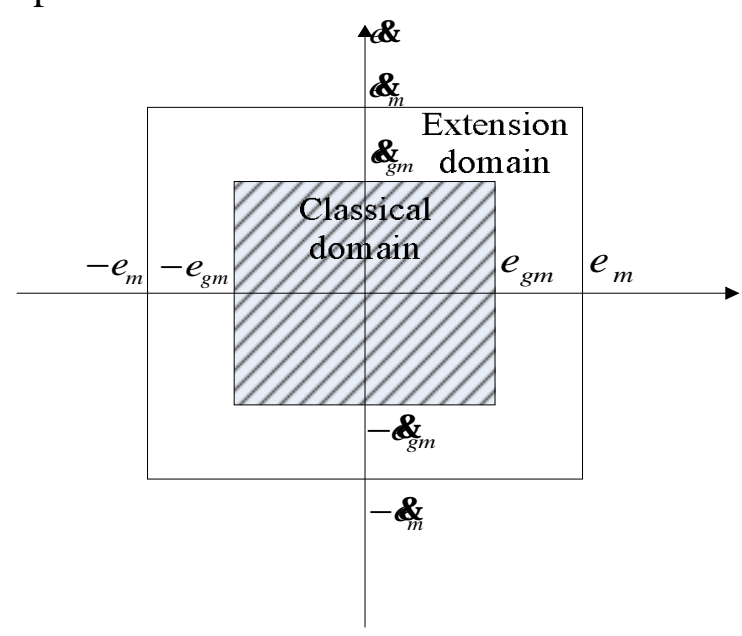

Fig.2 Extension set of feature description

\section{Correlation Calculation}

Setting the origin of the feature plane is $S_{0}(0,0)$, and $M_{0}=\sqrt{e_{g m}^{2}+\&_{g m}^{2}}, M_{-1}=\sqrt{e_{m}^{2}+\&_{m}^{2}}$, the correlation of any point within the feature plane is defined as

$$
K(s)= \begin{cases}1-\left|S S_{0}\right| / M_{0} & S \in R_{g y} \\ \left(M_{0}-\left|S S_{0}\right|\right) /\left(M_{-1}-M_{0}\right) & S \notin R_{g y}\end{cases}
$$

Where, $\left|S S_{0}\right|=\sqrt{k_{1} e^{2}+k_{2} \&}, R_{g y}$ is the classical domain shown in Figure $2, k_{1}, k_{2}$ are feature coefficients.

\section{Division of Measurement Mode}

The correlation Ks reflects the degree of association between the feature state of the system and the extension set of feature state, which can be divided into three measurement modes.

(1)Measurement mode $M_{1}=\{S \mid K(s) \geq 0\}$, the corresponding characteristic state belong to the classical domain. In this case, the feature quantity located in the acceptable range of control volume requirement .

(2)Measurement mode $M_{2}=\{S \mid-1 \leq K(s)<0\}$, the corresponding characteristic state belong to the extension domain. In this case, the characteristic quantity does not fit the control requirements, but it can be controlled by changing the value of the variable leaving the feature state within the acceptable range.

(3)Measurement mode $M_{3}=\{S \mid K(s)<-1\}$, the corresponding characteristic state belong to the Non-domain. In this case, it can not be controlled by changing the value of the variable to make the feature state within the acceptable range, then we need to change control variables, namely, controlled variables and control variables pair again.

\section{Control Strategy}

According to the measurement model of current system, adopting the corresponding control strategy[5].

(1)For measurement mode $M_{1}$, due to the feature quantity is within the control target range, so the output of the controller remains unchanged, namely to maintain the value before the moment of sampling. 
(2)For measurement mode $M_{2}$, based on extension control strategy, the output of the controller is

$$
u(t)=y(t) / k_{c}+k_{e} K(s)[-\operatorname{sign}(e)]
$$

Where, $\operatorname{sign}(e)=\left\{\begin{array}{cc}1 & e>0 \\ 0 & e=0 \\ -1 & e<0\end{array}\right.$

(3)For measurement mode $M_{3}$, the output of the controller takes the maximum $U_{m} \operatorname{sign}(e)$.

\section{Design of HVDC Extension additional frequency controller}

The HVDC supplementary frequency controller is mainly based on the characteristics of HVDC short time overload capacity and fast controllability characteristics that can be quickly adjusted as required, when the system is disturbed, DC power can be adjusted quickly according to the requirements, reducing the imbalance between turbine output and HVDC transmission power, thereby improving the stability of the system. When the system is disturbed to cause frequency variations, the additional controller takes the system frequency as a controller input signal to produce a DC modulation amount $\Delta P_{0}$, added to the main DC control module to adjust the DC power, suppressing the frequency fluctuation of the system, damping system shocks, and

$$
\Delta P_{0}=\left\{\begin{array}{lc}
0 & K(s) \geq 0 \\
f / k_{c}+k_{e} K(s)[-\operatorname{sign}(e)] & -1 \leq K(s)<0 \\
\Delta P_{m} \operatorname{sign}(e) & K(s)<-1
\end{array}\right.
$$

\section{Simulation and Analysis}

For the four-machine two-area system model, simulation and analysis of the additional frequency controller is conducted by PSCAD. The system is shown in Figure 3, two AC systems are connected via a DC line, and the rated capacity of the DC line is $1200 \mathrm{MW}$, the rectifier adopts constant current control,while the inverter adopts constant extinction angle control.

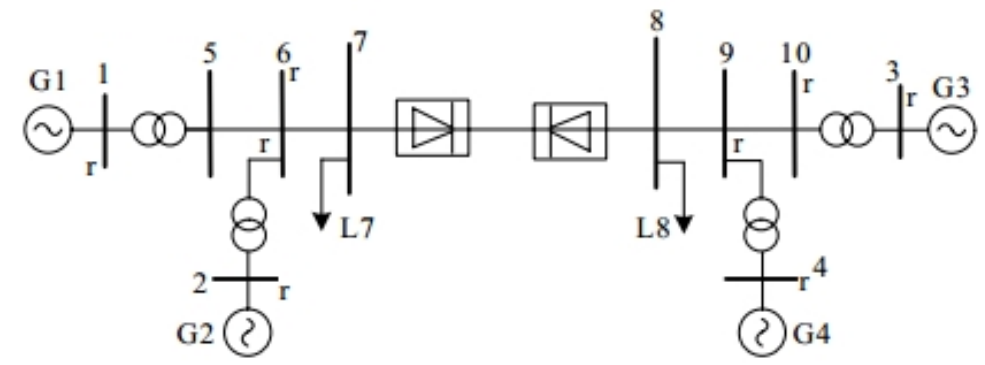

Fig.3 The system to be researched

The left area of the DC line is Zone1,while the right area is Zone2, setting removal load of 100 MW at L8 region 2, at this point, there are excess of active power in region2, and the frequency increases. The original frequency response and the frequency response after added the HVDC controller are shown in Figure 4. 


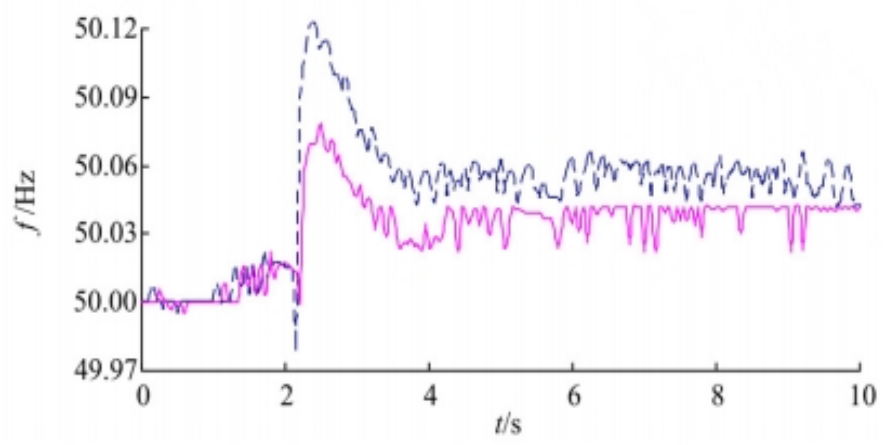

Fig.4 Frequency response of cutting load of area 2

It can be seen from the comparison result, without adding additional frequency controller, the active power imbalance will lead to a greater frequency fluctuations, and after adding additional frequency controller, frequency fluctuations eased markedly.

Setting three phase is shorted to ground at area 2 bus 8 , recovered after $0.2 \mathrm{~s}$, the change of system frequency is shown in Figure 5, obviously,after the additional frequency controller designed was added, the frequency fluctuations of defective area eased markedly, limited to a maximum frequency fluctuations in less than $50.15 \mathrm{~Hz}$, and tend to be stable faster, improved the frequency stability of the system significantly.

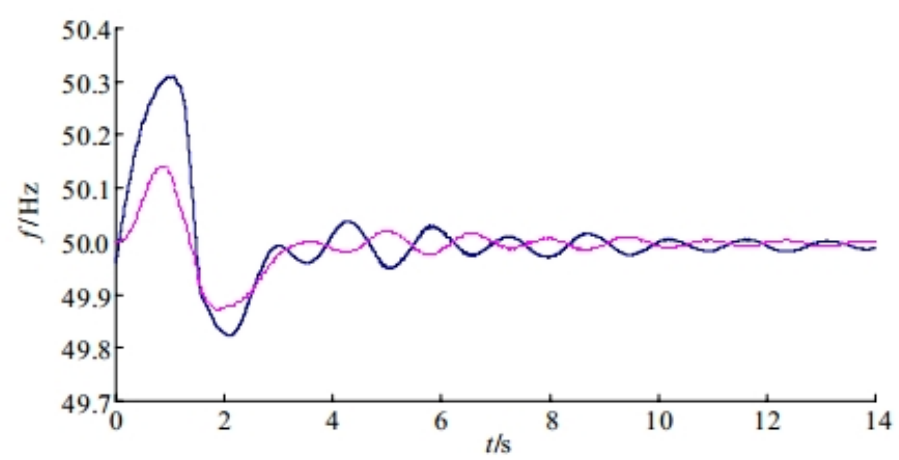

Fig.5 Frequency response of short circuit of area 2

\section{Conclusions}

In this paper, a kind of additional DC frequency controller based on extension control was designed, which has the advantages of simple structure, easy to be implemented. For the four-machine two-area system model, the simulation and analysis found that the controller can effectively play the role of system frequency fluctuation under the stable perturbation and the accident state.

\section{References}

[1] MA Yulong, SHI Yan, YIN Weiyang, et al. Additional control strategy for islanded AC system at sending terminal of HVDC[J]. Power System Technology, 2006,30(24): 22-25.

[2] SHAO Zhenxia, Li Xingyuan. HVDC emergency power support control based on a adaptive fuzzy controller[J]. Automation of Electric Power Systems, 2002, 26(5): 1-4.

[3] WANG Mingdong, LIU Xianlin, YU Jilai. Extension excitation controller of synchronous generator[J]. Electric Machines and Control, 2008,12(1):1-4.

[4] WANG Mingdong, LIU Xianlin, YU Jilai. Extension-based control strategy for governor of hydro-generator[J]. Journal of Hydroelectric Engineering, 2009,28,(4):171-175,186.

[5] CAI Wen, The extension set and non-compatible problem, Science Exploration (m Chinese), 1983, 3(1); 83. 